\title{
Feasibility Study of UAV Implementation in Route Surveying
}

\author{
Hazry Desa ${ }^{1}$, Muhammad Azizi bin Azizan ${ }^{1}$, Mohamad Syafiq Abdul Khadir ${ }^{1,}$, Muhammad Safwan Suhaimi ${ }^{1}$, \\ Noor Zulaiha Ramli ${ }^{1}$, Zainudin Hat ${ }^{2}$ \\ ${ }^{1}$ Centre of Excellence for Unmanned Aerial System (COEUAS), Universiti Malaysia Perlis (UniMAP), Kangar, Perlis, Malaysia \\ ${ }^{2} I P$ Fokus Sdn Bhd, Bandar Baru Bangi, Selangor, Malaysia
}

\section{ARTICLE INFO}

\section{Article History}

Received 21 November 2018 Accepted 02 December 2018

Keywords

UAV

engineering work

land survey

drone

\begin{abstract}
Unmanned Aerial Vehicle (UAV) are widely used in numerous field and the technology keeps growing. Generally, conventional method used in data collection for engineering work is tedious and requires a lot of manpower. This research focuses on the investigation of the suitability of UAV for route survey. Three stages involved: data collection, data processing and data analysis. By using Kama Beta, the data was collected. For data processing, Pix4D Mapper is used for point cloud and AutoCAD 2014 for the analysis stage. The result is mainly focused on extracting the road profile and test the point cloud data using Root Mean Square Error (RMSE). The result from both methods, conventional and UAV from the point cloud data using RMSE show only the small difference, with only $6.67 \%$ from total 60 points out of tolerance.
\end{abstract}

(C) 2019 The Authors. Published by Atlantis Press SARL.

This is an open access article distributed under the CC BY-NC 4.0 license (http://creativecommons.org/licenses/by-nc/4.0/).

\section{INTRODUCTION}

Engineering survey is a survey undertaken for the purpose of obtaining information essential to the planning of an engineering project. Engineering survey provides accurate and reliable dimension data through the construction process from the base topographical survey, cross-section and ground modelling information to cut and fill calculation, dimensional control to setting-out on-site and finished as-built drawing [1].

Route surveying is comprised of all survey operation required for designing and construction of engineering works such as highways, pipelines, canals or railroads. Survey operations in route survey include topographic survey, cross-section and longitudinal section [2].

Unmanned Aerial Vehicles (UAVs), also known as "drones", are among the most important technological advances that have been introduced to the land surveying industry in quite some time. It can be piloted by remote and can take detailed survey information while simultaneously transmitting that data back to the head office. UAVs create a highly accurate map and provide valuable data to companies and individuals who are considering major projects on large areas of land. Research about the reliability of these techniques to be used in topographic mapping was conducted in land survey method [3].

\section{METHODOLOGY}

\subsection{Planning and Preparation}

Planning and preparation are needed before the data collection. Planning and preparation cover the scope of work such as planning

"Corresponding author.Email: msyafiq@unimap.edu.my for the area of the survey and the preparation for the instrument and software used in this project. Good planning and preparation enable to ensure efficiency and maintaining a high quality of the data collection in this study. Furthermore, proper planning and preparation help in saving time and cost as time increase, the cost will increase as well.

\subsection{Data Collection}

Data collection for this study is focused on two methods. The first method of data is used as a conventional method by total station. Besides that, the second method for this study used a new method by UAV.

\subsection{Data Processing}

Data processing for this study refers to process the laser and the conventional method. Data processing for a conventional method uses the Civil Design \& Survey software. Besides that, for the UAV processing used Pix4D Mapper. Both use AutoCAD as data analysis processing.

\section{RESULTS AND ANALYSIS}

\subsection{Camera Calibration}

The camera calibration is done before doing the flight planning. The purpose of camera calibration is to determine the focal length, principal point, and lens distortion from the camera. This process was carried out to figure out the unstable element in the camera like interior orientation and lens distortion parameters [4]. 
In this project, the camera calibration is processed using PhotoModeler Software. This software is an automatic lens calibration, which uses A1 paper sheet as a calibration target. It supports estimations of the full camera calibration matrix, including non-linear distortion coefficient.

To check the accuracy of the camera calibration results, the total final error must be checked. In both methods, the data sets are not the same. For lab calibration, the data set is the grid of the pattern and for filed calibration are the 67 targets points. According to PhotoModeler tutorial, a value $<1.0$ pixel indicates a good calibration and very good calibrations can have a final total error smaller than 0.4 pixels (www.photomodeler.com). In this case, the lab calibration has a final error of 1.940 pixels [7].

The total error was a bit higher than the recommended. The field calibration has a total error of 0.282 pixels, which is assumed to be a very good calibration project. Also checking the marking residuals is a good way to test the calibration quality [4]. PhotoModeler tutorial recommended having the largest marking residual $<1.0$ pixel [3]. In both cases, the largest marking residuals are $<1.0$ pixel. The lab calibration has 0.723 pixels and the field calibration 0.700 pixels (Table 1 ).

The accuracy of field calibration was also checked to compare the Global Positioning System (GPS) coordinates of the targets with the coordinates obtained with PhotoModeler. This software only needs three control points to change from relative to absolute coordinates. For this process, the targets number one, two and four were used. The planimetric and the altimetric Root Mean Square (RMS) were calculated. The planimetric RMS was $0.028 \mathrm{~m}$ and the altimetric $0.026 \mathrm{~m}$, which is really small and indicates the accuracy level of the project [7].

\subsection{Georeferencing}

Ground Control Point (GCP) and Verification Point (VP) are very important data that are used to involve in aerial triangulation phase, where it can be identified on the ground features and can be georeferenced with the aerial image used in geometric correction of the distorted image [3]. Therefore, pre-marking for GCP's and VP's must be carried out before a flight mission. This is because the processing of the tie point image could be more efficient and accurate. The coordinates of $x, y$ and $z$-value that were observed using levelling method (land survey).

Figure 1 shows the strategic location of placement six (6) GCP's. The location of GCP was in Chuping, Perlis, Malaysia. Each of GCP is observed by GPS equipment (Topcon - GR5) using Static Method. The reason of this method selection is because this method is the most widely used differential technique for control and geodetic surveying, involved $1 \mathrm{~h}$ of observation to resolve the integer ambiguities between the satellite and the receiver. By using this method, the accuracies in the sub-centimeter range can be obtained.

Table 1 Total final error and residuals of the camera

\begin{tabular}{lcc}
\hline & $\begin{array}{c}\text { Lab } \\
\text { calibration }\end{array}$ & $\begin{array}{c}\text { Field calibration } \\
\mathbf{5 0} \mathbf{~} \text { } \text { flight high })\end{array}$ \\
\hline Final total error (pixel) & 1.940 & 0.282 \\
Largest marking residual (pixel) & 0.723 & 0.700 \\
Overall RMS (pixel) & 0.245 & 0.341 \\
\hline
\end{tabular}

\subsection{Digital Surface Model}

The aerial image was taken from above with 14 min flight duration. To achieve this project the overlap is $80 \%$, sidelap is $70 \%$ and the altitude is $110 \mathrm{~m}$. This overlapping and flight altitude is required to avoid potential missing areas of coverage and to ensure good coverage. Based on the data that has been processed using Pix4D software, produced an Orthophoto and Digital Surface Model (DSM) in raster as shown in Figures 1 and 2.

Figure 3 shows the sample result road profile of UAV and TS data for $\mathrm{CH} 1$ to $\mathrm{CH}$ 10, which is located in Chuping, Perlis, Malaysia. Based on Figure 3, the red color refers to the highest elevation while the soft blue color refers to the lowest elevation [5]. Therefore, it can clearly be seen that the UAV data gives a greater number of point compared with TS data. Besides that, the profile generated by using UAV is denser than the TS data due to the great number of point [6].

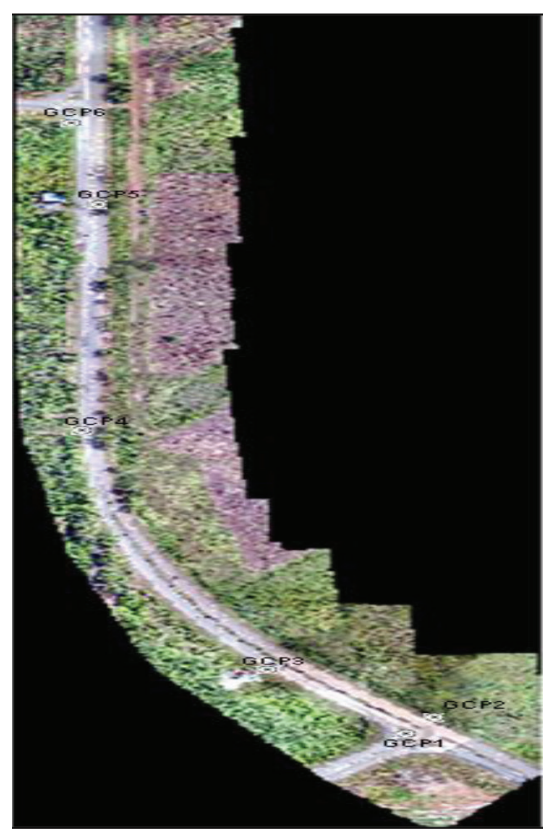

Figure 1 Location of GCP in UAV images in Chuping, Perlis, Malaysia (Orthophoto).

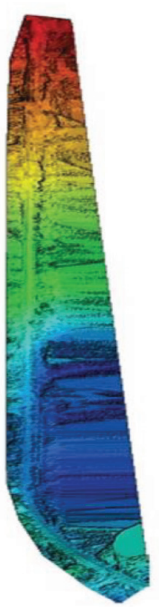

Figure 2 DSM of the project area (Location: Chuping, Perlis, Malaysia). 


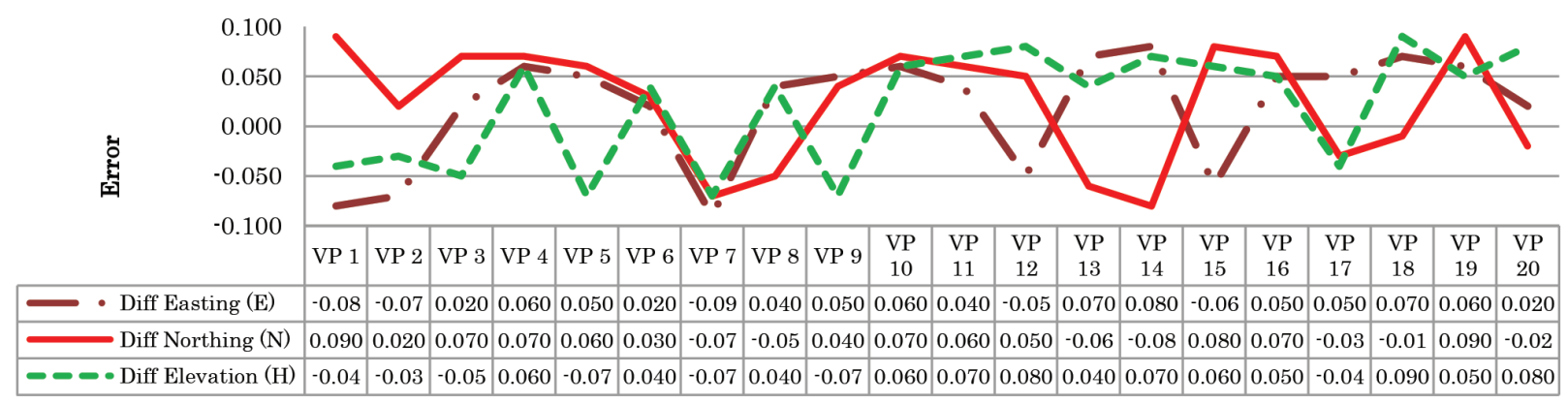

Figure 3 RMSE error verification points.

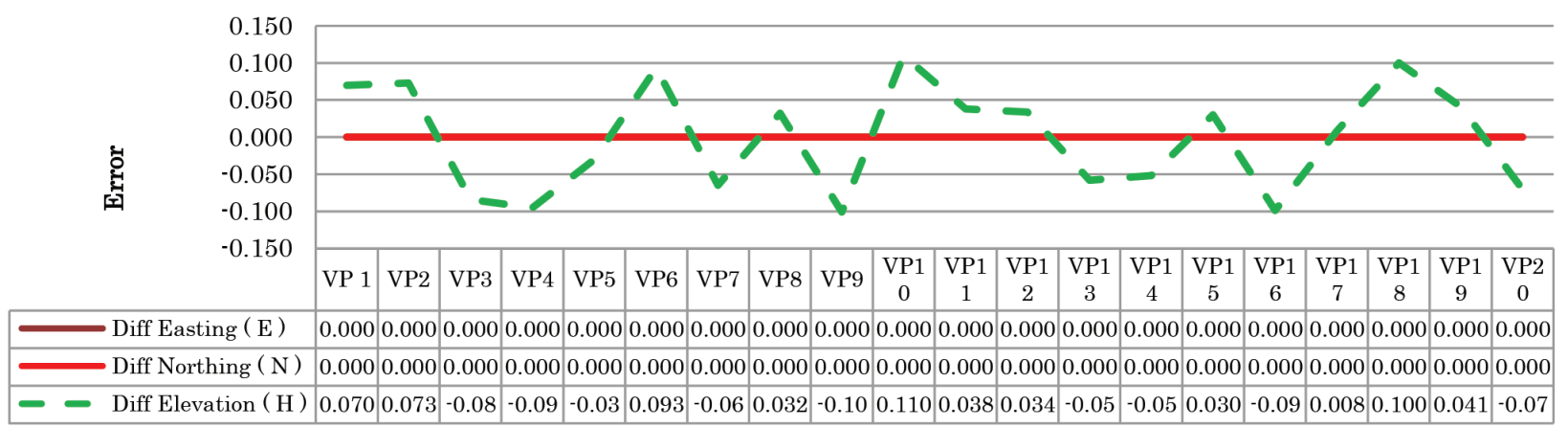

Figure $4 \mid$ RMSE error cross-section.

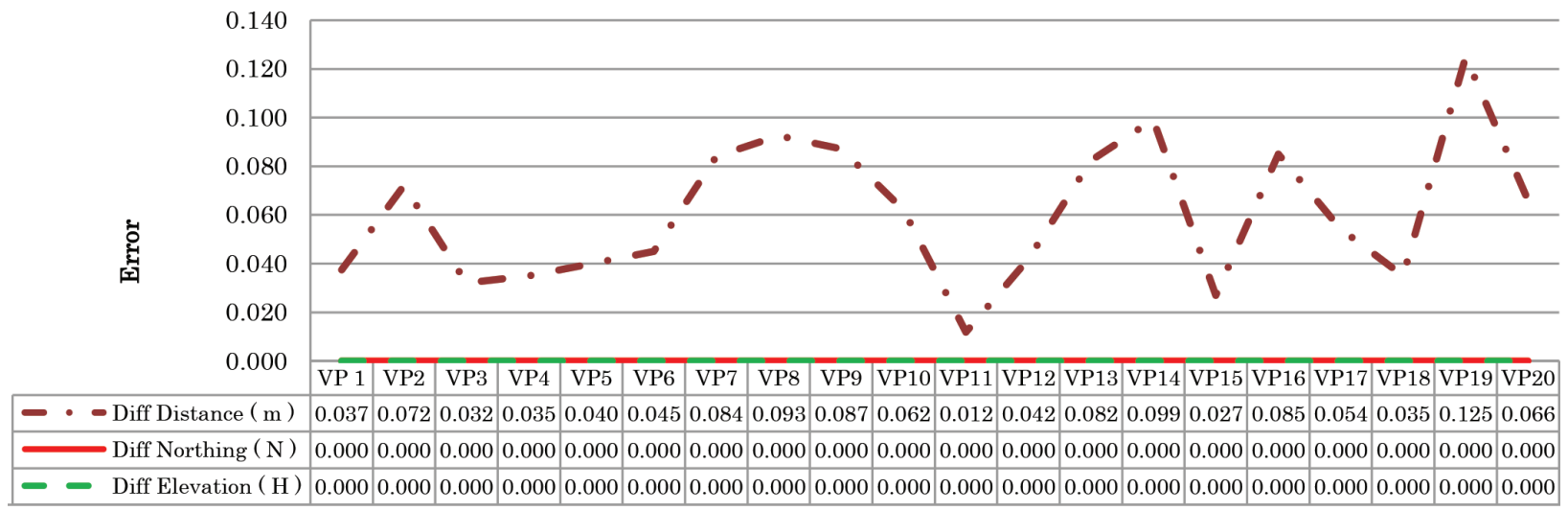

Figure 5 Residual error XY.

\subsection{Statistical Analysis}

The road profiles are tested with RMS Error (RMSE) which is done by analyzing the comparison of differences between and within the group. The road profile is statistically tested by using the RMSE test at each chainage to ensure that the data of UAV and TS is significant to each other.

Root mean square error is the standard deviation of the residuals (prediction errors). Residuals are a measure of how far from the regression line data points are; RMSE is a measure of how to spread out these residuals are. In other words, it tells you how concentrated the data is around the line of best fit. RMSE is commonly used in climatology, forecasting, and regression analysis to verify experimental results [5].

The formula (1) is:

$$
\operatorname{RMSE}_{f o}=\left[\sum_{i=1}^{N} \frac{\left(z_{f_{i}}-z_{o_{i}}\right)^{2}}{N}\right]^{\frac{1}{2}}
$$

where $\Sigma=$ summation of, $\left(z_{f i}-z_{o i}\right)^{2}=$ (differenced, squared), $N=$ sample size.

There are three types of sample tested using RMSE formula, verification point, positioning and elevation. Values from the real measurement are compared with the value from the $3 \mathrm{D}$ modelling to get the error of every sample.

Based on Figure 3, it can be seen that the graph values of RMSE VP are for minimum and maximum errors. All the VPs are under tolerance which is below $\pm 0.10 \mathrm{~m}$. Based on Figure 4 , it can be seen that the graph values of RMSE Cross Section are for minimum and maximum errors are for Different Elevation (H). The best result is $\pm 0.03 \mathrm{~m}$ (VP5). VP9, VP10, and VP18 are out of tolerance which is above $\pm 0.10 \mathrm{~m}$.

The values of RMSE XY for minimum and maximum errors can be referred to Figure 5. All the XY are under tolerance which is below $\pm 0.10 \mathrm{~m}$ except at VP19 with value $\pm 0.125 \mathrm{~m}$. These results might be affected by image matching algorithm that was used in the same software during image processing. The error was usually 
caused by flying height during image acquisition, image matching during image processing and motion movements such as $\omega, \pi$, and $\kappa[6]$.

\section{CONCLUSION}

As a conclusion, the three objectives of this study has been achieved. The first objective is to process image capture from UAV. The most important procedure for route survey work is data collection. Planning and preparation in this study focusing on planning for a number of GCP and flight planning. Number of GCP depends on a number of flight planning where need minimum visible from the aerial view and location of GCP. After that, the procedure for route survey by using UAV need to focus on processing the point cloud data. Starting with data registration point cloud data uses minimum of three images that visible each other. Then, the next process is point cloud densification using PIX4D Mapper software. Then, the next process produces DSM and Orthophoto. In this research, two methods were applied, land survey and UAV survey.

A land survey is used as a benchmark for this research. Point cloud data produce in $2 \mathrm{D}$ data with $3 \mathrm{D}$ coordinate which can use to generate road profile for route survey. The third objective for this study is to analyse the accuracy between land survey data and UAV data. Series of test and analysis have been conducted in terms of the reliability of UAV data in completing a route survey. The reliability of UAV data shows in quantitative assessment by comparing result road profile from UAV and TS method.

\section{CONFLICTS OF INTEREST}

The authors declare they have no conflict of interest.

\section{ACKNOWLEDGMENTS}

The author acknowledges International Conference on Artificial Life and Robotics (ICAROB) for giving the opportunity to join this conference. High appreciation to UAS research team for excellent contribution. Special gratitude to IP Fokus Sdn Bhd for full support in completing this project.

\section{REFERENCES}

[1] A. Ahmad, K.A. Hashim, A.M. Samad, Aerial mapping using high resolution digital camera and unmanned aerial vehicle for Geographical Information System, 2010 6th International Colloquium on Signal Processing \& its Applications, IEEE, Mallaca City, Malaysia, 2010, pp. 1-6.

[2] H. Eisenbeiss, A mini unmanned aerial vehicle (UAV): system overview and image acquisition. International workshop on processing and visualization using high-resolution imagery, institute for geodesy and photogrammetry, ETH, Hoenggerberg, Zurich, Switzerland; 2004.

[3] J. Everaerts, The use of unmanned aerial vehicles (UAVs) for remote sensing and mapping. International Archives of The Photogrammetry, Remote Sensing and Spatial Information Sciences (ISPRS Archives), Beijing, 2008, pp. 1187-1191.

[4] V. Peterman, M. Mesarič, Land survey from unmanned aerial vehicle. International archives of the photogrammetry, remote sensing and spatial information sciences, XXXIX-B1 (2012), 447-451.

[5] M. Pérez, F. Agüera, F. Carvajal, Digital camera calibration using images taken from an unmanned aerial vehicle. International Archives of the Photogrammetry, Remote Sensing and Spatial Information Sciences, XXXVIII-1/C22, Conference on Unmanned Aerial Vehicle in Geomatics, Zurich, 2011, pp. 167-171.

[6] A.M. Samad, N. Kamarulzaman, M.A. Hamdani, T.A. Mastor, K.A. Hashim, The potential of Unmanned Aerial Vehicle (UAV) for civilian and mapping application. 2013 IEEE 3rd International Conference on System Engineering and Technology, IEEE, Shah Alam, Malaysia, 2013.

[7] H. Desa, M.A. Azizan, S. Suhaimi, Z. Ramli, M.S. Abdul Khadir, Z. Hat, Feasibility Study of UAV Implementation in Route Surveying, The 2019 International Conference on Artificial Life and Robotics (ICAROB2019), 24 (2019), 712-716.

\section{Authors Introduction}

\section{Prof. Ts. Dr. Hazry Desa}

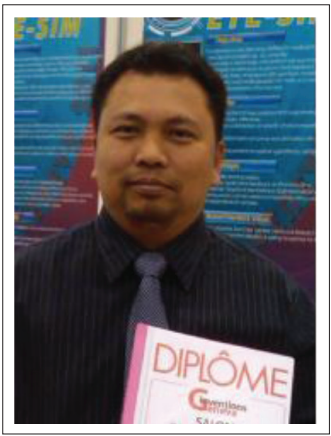

$\mathrm{He}$ is a Director of Center of Excellence for Unmanned Aerial System at University of Perlis (UniMAP). He has published many publications and filled patents on the application of UAV for crop spraying and monitoring. He leads the educational program and organized several UAS related conference in Malaysia. He is a member of IEEE.

\section{Mr. Ts. Muhammad Azizi bin Azizan}

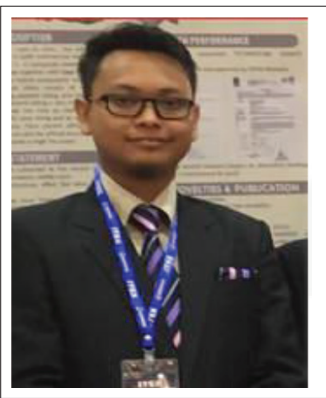

He received his MSc degree from University of Technology Mara (UiTM) in Integrated Project Management. He is a Non-Executive Group Director, Aurum Group and Vice President (Malaysia Global Economic Chamber of Commerce). Registered as CIOB UK, CIBSE UK, HRDF Malaysia and RISM Malaysia. Nature of research are Property, Construction, Consultation, Manufacturing and Supply. 


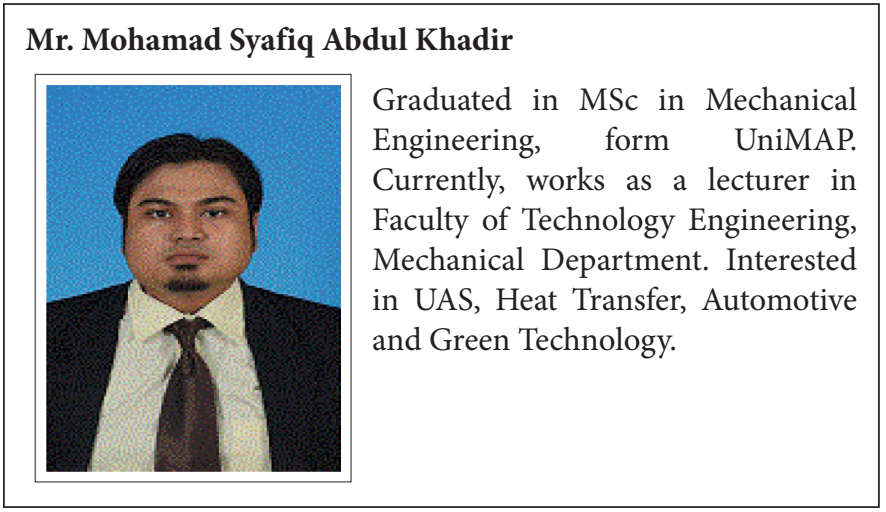

\section{Mr. Muhammad Safwan Suhaimi}

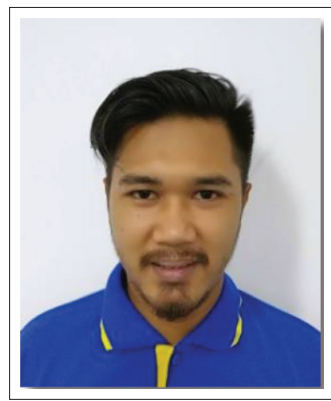

Graduated from UiTM Arau in 2014. Works as Geospatial Engineer and Research Assistant under Center of Excellence for Unmanned Aerial System (COEUAS) UniMAP.

\section{Noor Zulaiha Ramli}

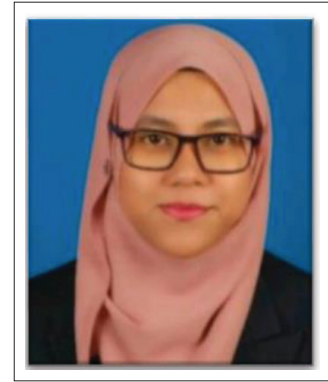

Graduated from UiTM Arau in 2014.

Works as Geospatial Engineer and

Research Assistant under Center of

Excellence for Unmanned Aerial

System (COEUAS) UniMAP.

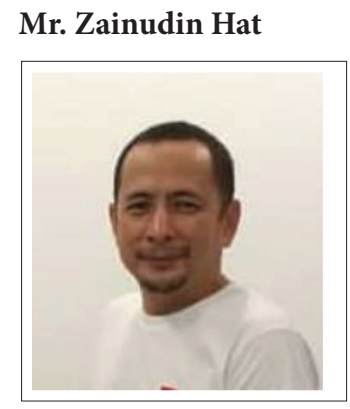

The owner of IP Fokus Sdn Bhd Actively involved in research for Unmanned Aerial System (UAS), Building surveying and Construction. 